\title{
EVALUATION OF PROCESS PARAMETERS FOR ENZYMES PRODUCTION FROM TRICHODERMA SP. SH2 USING PINE NEEDLES BIOMASS UNDER SOLID STATE FERMENTATION
}

\author{
Dimple Tanwar, Nivedita Sharma* and Nisha Sharma
}

\begin{abstract}
Microbiology laboratory, Department of Basic Sciences, Dr Y S Parmar university of Horticulture \& Forestry, Nauni- Solan, H.P.
\end{abstract}
Email : niveditashaarma@yahoo.co.in

\begin{abstract}
Various optimization steps by using classical one factor at a time approach were carried out to make the production of hydrolytic enzyme cost effective and commercially viable from a potent fungal strain Trichoderma sp. SH2 under solid state fermentation using pine needles biomass as a substrate. The parameters i.e. temperature, moisture: substrate ratio, incubation days and $\mathrm{pH}$ were studied. After optimization appreciable amount of enzymes and reducing sugars i.e. laccase $(3.75 \mathrm{U} / \mathrm{g})$, cellulase $(27.45 \mathrm{U} / \mathrm{g})$, xylanase $(350.17 \mathrm{U} / \mathrm{g})$ and reducing sugars ( $36.90 \mathrm{mg} /$ ) has been achieved by using untreated pine needle biomass.
\end{abstract}

Keywords: Enzymes, Pine needles, Fermentation, Trichoderma sp. SH2 


\section{Introduction}

Lignocellulosic biomass is a promising renewable resource for attaining value added products. (Acharya et al., 2010). Many microorganisms are capable of producing a variety of lignocellulolytic enzymes i.e. laccase, cellulase and xylanase which are responsible for the biodegradation of lignocellulosic wastes (Barakat et al., 2014). Biotechnology operations for enzyme production are potential alternative proposition for the future (Mishra et al., 2016). Moreover, these wastes may contain significant concentrations of soluble carbohydrates, which are efficient in the production of ligninolytic enzymes (Elisashvili et al., 2009). These enzymes are strongly inhibited by the presence of cellobiose (Massadeh et al., 2001). They are produced by living organisms to increase the rate of a chemical reactions required for life (Li et al., 2012). Being rich in cellulase, xylanase and laccase, lignocellulolytic fungi serve as a substrate for biodegradation of pine needles for the production of biofuels besides its safe disposal helping to solve the burning issue of global warming (Nagraj et al., 2014). Therefore, the aim of this study was to investigate the performance of the potential strain Trichoderma sp. SH2 isolated from rotten wood in producing ligninocellulases (laccase, cellulase and xylanase) using pine needles as substrates under sold state fermentation mode.

\section{Materials and Methods}

Collection of pine needles
The lignocellulosic pine needles were collected from different coniferous forests

of Himachal Pradesh i.e. Solan, Sirmour, Kasauli, Narag, Nauni, Sultanpur and Dharampur and chipped in to $2.0 \mathrm{~mm}$ mesh size.

\section{Microwave pretreatment}

Pine needle biomass was subjected to microwave irradiation at $600 \mathrm{~W}$ for $3 \mathrm{~min}$.

\section{Enzymes production and extraction (Bollag and Edelstein, 1991)}

$10 \%$ of inoculum of Trichoderma sp. SH2 was added to the 5 gram of biomass and kept for incubation in the static condition. After incubation, $50 \mathrm{ml}$ of sodium citrate buffer $(0.005 \mathrm{M}, \mathrm{pH} 5.5)$ was added in 250 $\mathrm{ml}$ Erlenmeyer flask. The contents were kept in the shaker for $1 \mathrm{~h}$ at $120 \mathrm{rpm}$ and then filtered and centrifuged at 10,000 rpm for $10 \mathrm{~min}$ at $4^{\circ} \mathrm{C}$. The supernatant was collected for further studies.

\section{Effect of Moisture level}

Different substrate: moisture ratios i.e. 1:2, $1: 3,1: 4,1: 5$ and 1:6 were studied for enzyme production at $30^{\circ} \mathrm{C}$ for 7 days.

\section{Effect of temperature}

Temperature ranges i.e. $20^{\circ} \mathrm{C}, 25^{\circ} \mathrm{C}, 30^{\circ} \mathrm{C}$, $35^{\circ} \mathrm{C}, 40^{\circ} \mathrm{C}$ and $45^{\circ} \mathrm{C}$ for 7 days.

\section{Effect of Incubation time}

Different incubation days ranging from 3,5 , $7,10,12$ days at 30 were studied. 
$\mathrm{pH}$ ranging from 4.5, 5.0, 5.5, 6.0 and 6.5 were used in the study.

\section{Enzyme Assays}

\section{Laccase assay}

Laccase assays were performed as per Rehan et al., method (Rehan et al., 2016).

\section{Cellulase assay}

The sub-enzymes CMCase and FPase were measured by following standard method of Reese and Mandel (Reese and Mandel, 1963). For $\beta$-glucosidase activity the standard method of Berghem and Petterson was followed (Berghem and Petterson, 1973).

\section{Xylanase Assay}

Quantitative assays were performed for xylanase assay by applying Miller's method (Miller, 1959).

\section{Reducing sugars}

Reducing sugars were estimated by using DNSA method (Miller, 1959).

\section{Protein assay}

Protein estimation was done by using standard protocol of Lowry et al., 1951 (Lowry et al., 1951).

\section{Results and Discussion}

\section{Effect of Temperature}

Effect of temperature on laccase, cellulase, xylanase and reducing sugars as well as biodegradation index from Trichoderma sp. SH2 was studied using untreated and pretreated pine needles biomass. Maximum laccase activity was observed at $30^{\circ} \mathrm{C}$ i.e. 2.82 with specific activity i.e. $0.01 \mathrm{U} / \mathrm{mg}$ respectively, whereas least laccase enzyme production for was found at $45^{\circ} \mathrm{C}$ i.e. $0.60 \mathrm{U} / \mathrm{g}$ with specific activity i.e. $0.02 \mathrm{U} / \mathrm{mg}$ respectively (table 1). Maximum cellulase activity was shown at $30^{\circ} \mathrm{C}$ i.e. $23.10 \mathrm{U} / \mathrm{g}$ with $0.82 \mathrm{U} / \mathrm{mg}$ specific activity whereas least cellulase production was found at $45^{\circ} \mathrm{C}$ i.e. 12.39 $\mathrm{U} / \mathrm{g}$ with $0.50 \mathrm{U} / \mathrm{mg}$ specific activity respectively (table 1). Maximum xylanase activity for untreated biomass is $268.97 \mathrm{U} / \mathrm{g}$ and least xylanase activity of $106.89 \mathrm{U} / \mathrm{g}$ with specific activity $4.39 \mathrm{U} / \mathrm{mg}$ was observed at $45^{\circ} \mathrm{C}$ as shown in table 1.

Table 2 depicted the effect of temperature on pretreated pine needles biomass. Maximum laccase activity i.e. $2.72 \mathrm{U} / \mathrm{g}$ with specific activity i.e. $0.09 \mathrm{U} / \mathrm{mg}$ was observed at $30^{\circ} \mathrm{C}$ and least activity was recorded at $45^{\circ} \mathrm{C}$ i.e. $0.13 \mathrm{U} / \mathrm{g}$. The maximum cellulase activity was observed at $30^{\circ} \mathrm{C}$ i.e. $20.70 \mathrm{U} / \mathrm{g}$ with specific activity i.e. $0.71 \mathrm{U} / \mathrm{mg}$ specific activity (table 2). On the other hand, the maximum xylanase activity was observed at $30^{\circ} \mathrm{C}$ i.e. 265.63 $\mathrm{U} / \mathrm{g}$ with $9.22 \mathrm{U} / \mathrm{mg}$ specific activity and least activity was reported at $45^{\circ} \mathrm{C}$ i.e. 97.65 (table 2). Microorganisms grow slowly at a temperature below or above the normal temperature because of reduced rate of cellular production. The reduction in enzyme activity at higher temperature could be due to denaturation of enzymes. Maximum enzyme activity at optimum temperature may be due to the faster metabolic activity and increase in protein content and extracellular enzyme production in culture supernatant (Sharma and Sharma, 2016). 
Effect of substrate: moisture ratio

The data revealed the difference in laccase, cellulase and xylanase production at different moistening agent i.e. 1:2, 1:3, 1:4, 1:5 and 1:6. Maximum laccase activity using untreated pine needles biomass was observed i.e. 3.45 $\mathrm{U} / \mathrm{g}$ with specific activity $0.10 \mathrm{U} / \mathrm{mg}$ at $1: 4$ whereas least laccase production was found at 1:2 i.e. $0.89 \mathrm{U} / \mathrm{g}$ (Table 3). The maximum cellulase was found at 1:4 i.e. $27.90 \mathrm{U} / \mathrm{g}$. Whereas least cellulase production was found i.e. $20.40 \mathrm{U} / \mathrm{g}$ at 1:6 substrate moisture level. The maximum xylanase activity was $279.30 \mathrm{U} / \mathrm{g}$ with specific activity $8.54 \mathrm{U} / \mathrm{mg}$ at $1: 4$ and least xylanase activity was found i.e. $234.75 \mathrm{U} / \mathrm{g}$ with specific activity $8.28 \mathrm{U} / \mathrm{mg}$ at 1:6.

Table 4 depicted the effect of substrate: moisture ratio on pretreated pine needles biomass. Maximum laccase activity i.e. $3.16 \mathrm{U} / \mathrm{g}$ with specific activity of $0.12 \mathrm{U} / \mathrm{mg}$ at 1:4 substrate moisture level was observed and least activity was recorded at $1: 2$ i.e. $0.39 \mathrm{U} / \mathrm{g}$. The maximum cellulase activity was found at 1:4 i.e. $27.00 \mathrm{U} / \mathrm{g}$ with specific activity of $1.04 \mathrm{U} / \mathrm{mg}$ and least activity was observed i.e. $18.75 \mathrm{U} / \mathrm{g}$ with specific activity 0.80 at 1:2 substrate moisture level. In case of xylanase, the maximum xylanase activity using pretreated pine needles biomass at 1:4 substrate moisture level i.e. $277.95 \mathrm{U} / \mathrm{g}$ with specific activity $10.77 \mathrm{U} / \mathrm{mg}$ at $1: 5$ and least activity was reported at 1:2 substrate moisture i.e. $228.15 \mathrm{U} / \mathrm{g}$.

As concerned the reducing sugar production, maximum reducing sugars from untreated pine needle biomass was $26.10 \mathrm{mg} / \mathrm{g}$ at 1:4 was observed while in pretreated pine needles biomass maximum reducing sugars of $32.65 \mathrm{mg} / \mathrm{g}$ at 1:4 was recorded (table 3 and 4). Moisture is essential for growth of microorganisms, therefore in the present study, depending upon the specific characteristics water activity $\left(a_{w}\right)$ requirement of all different selected strains, a variation in substrate: moisture ratio has been exhibited (Sharma et al., 2019).

\section{Effect of incubation time}

The different incubation days i.e. 3, 5, 7, 10 and 12 days were used for assessing the enzyme activity. Effect of incubation days on Trichoderma sp. SH2 for laccase enzyme production has been shown in table 5 and 6 . Maximum laccase activity was observed i.e. $3.45 \mathrm{U} / \mathrm{g}$ with specific activity 0.12 at 10 days, whereas least laccase enzyme production was found $0.27 \mathrm{U} / \mathrm{g}$ at 3 days with specific activity 0.02 $\mathrm{U} / \mathrm{mg}$.

The maximum cellulase activity for untreated biomass i.e. $27.45 \mathrm{U} / \mathrm{g}$ with specific activity $1.08 \mathrm{U} / \mathrm{mg}$ at 7 days, whereas least cellulase production was found i.e. $20.40 \mathrm{U} / \mathrm{g}$ with specific activity of $0.83 \mathrm{U} / \mathrm{mg}$ at 3 days. In case of xylanase enzyme, the maximum i.e. $293.10 \mathrm{U} / \mathrm{g}$ of xylanase was observed at 10 days and least xylanase activity was found i.e. 253.50 $\mathrm{U} / \mathrm{g}$ at 3 days.

Table 6 depicted the effect of incubation days on hydrolytic enzymes production using pretreated pine needles biomass. Maximum laccase activity was observed i.e. $3.30 \mathrm{U} / \mathrm{g}$ with specific activity i.e. 0.01 $\mathrm{U} / \mathrm{mg}$ at 10 day and least activity was found i.e. $0.30 \mathrm{U} / \mathrm{g}$ at 12 days. The 
maximum cellulase activity was found at 7 days i.e. $25.80 \mathrm{U} / \mathrm{g}$ at 12 days and least activity was observed i.e. $19.20 \mathrm{U} / \mathrm{g}$ at 12 days of incubation. Where as maximum xylanase activity was found at 7 days i.e. $289.50 \mathrm{U} / \mathrm{g}$ and least activity was observed i.e. $227.25 \mathrm{U} / \mathrm{g}$ at 3 days. In case of reducing sugar production, maximum reducing sugars from untreated pine needle biomass i.e. $36.75 \mathrm{mg} / \mathrm{g}$ were obtained after 7 days of incubation whereas in pretreated pine needles biomass maximum reducing sugars were observed $36.60 \mathrm{mg} / \mathrm{g}$. The decline in the activity afterwards could be due to various reasons. A decline in the enzyme afterwards may be because of proteolysis or due to depletion of nutrients available to the strain, causing a stressed microbial physiology resulting in an in activation of enzyme.

\section{Effect of $\mathrm{pH}$}

The data in Table 8 and 9 revealed the difference in laccase, cellulase and xylanase production at varying $\mathrm{pH}$ i.e. 4.5, $5,5.5,6,6.5 \mathrm{pH}$. Effect of $\mathrm{pH}$ for laccase enzyme production using untreated biomass has been shown in Table 7 . Maximum laccase activity was observed i.e. $3.75 \mathrm{U} / \mathrm{g}$ at $6.0 \mathrm{pH}$, whereas least laccase enzyme production was found i.e. $1.80 \mathrm{U} / \mathrm{g}$ at 4.5 . The maximum cellulase activity of $27.45 \mathrm{U} / \mathrm{g}$ with specific activity $1.03 \mathrm{U} / \mathrm{g}$ at $6.0 \mathrm{pH}$, whereas least cellulase production was found $21.45 \mathrm{U} / \mathrm{g}$ at 4.5 . As concerned xylanase activity, the maximum was observed at $5.5 \mathrm{pH}$ i.e. $350.17 \mathrm{U} / \mathrm{g}$ and least xylanase activity i.e. $209.26 \mathrm{U} / \mathrm{g}$ at 6.5 $\mathrm{pH}$ was observed. On the other hand,
Table 8 depicted the effect of $\mathrm{pH}$ on pretreated pine needles biomass. Maximum laccase activity was recorded i.e. $3.60 \mathrm{U} / \mathrm{g}$ with specific activity i.e. $0.14 \mathrm{U} /$ mg at $6.0 \mathrm{pH}$.

Table 8 also showed maximum cellulase activity for pretreated pine needles biomass i.e. $25.50 \mathrm{U} / \mathrm{g}$ with specific activity $0.97 \mathrm{U} / \mathrm{mg}$ at $6.0 \mathrm{pH} \mathrm{U} / \mathrm{mg}$ and least activity was observed i.e. $20.85 \mathrm{U} / \mathrm{g}$ at 4.5 $\mathrm{pH}$. Maximum xylanase activity of 335.25 $\mathrm{U} / \mathrm{g}$ with specific activity 12.99 was observed at $5.5 \mathrm{pH}$. On the other hand, maximum reducing sugars of $36.90 \mathrm{mg} / \mathrm{g}$ was achieved at $5.5 \mathrm{pH}$ (table 7) whereas in pretreated pine needles biomass, maximum reducing sugars i.e. $32.10 \mathrm{mg} / \mathrm{g}$ at $5.5 \mathrm{pH}$ were observed (table 8). Generally most of the fungi prefer to act favorably at lower $\mathrm{pH}$. In this study, optimum $\mathrm{pH}$ for maximum enzyme and sugar production had been noticed between 5 to 6.0 thus confirming the acidophilic nature of these fungi. Each microorganism possesses a specific $\mathrm{pH}$ range for its growth and activity. The extracellular $\mathrm{pH}$ has a strong influence on the pathways of metabolism and product formation by microorganism. Changes in the external $\mathrm{pH}$ alter the ionization of nutrient molecules and reduce their availability to the organism thus lowering their overall metabolic activity (Sharma and Sharma, 2016).

As the Fig ( 3 and 4 ) revealed the overall increase after optimization of different process parameters i.e. temperature, substrate: moisture ratio, incubation days and $\mathrm{pH}$ in untreated and pretreated pine needles biomass for extracellular enzymes 
activity i.e. $2.82 \mathrm{U} / \mathrm{g}$ to $3.75 \mathrm{U} / \mathrm{g}$ with \% increase of 75.20 (laccase), $23.10 \mathrm{U} / \mathrm{g}$ to $29.70 \mathrm{U} / \mathrm{g}$ with \% increase of 77.77 (cellulase), $268.97 \mathrm{U} / \mathrm{g}$ to $350.17 \mathrm{U} / \mathrm{g}$ with \% increase of 76.81 (xylanase) and 25.35 $\mathrm{mg} / \mathrm{g}$ to $36.90 \mathrm{mg} / \mathrm{g}$ with \% increase of 68.69 (reducing sugars). It showed the best enzyme activity in untreated pine needles biomass over pretreated biomass under
OFAT with percent increase 142.55 (cellulase), 109.26 (xylanase), 213.50 (laccase) and 140.18 (reducing sugars) therefore this potential strain Trichoderma sp. $\mathrm{SH} 2$ is highly recommended for biological pretreatment for lignocellulosic waste and for the production of industrially important enzymes. 


\section{J.Bio.Innov 9(6), pp: 1501-1519, 2020 |ISSN 2277-8330 (Electronic)}

Table1. Effect of temperature on enzymes production using untreated biomass

\begin{tabular}{|c|c|c|c|c|c|c|c|c|c|c|c|c|c|c|}
\hline $\begin{array}{l}\text { Temperature } \\
(\circ \mathrm{C})\end{array}$ & $\begin{array}{l}\text { Laccase } \\
\text { activity* }\end{array}$ & $\begin{array}{c}\text { Specific } \\
\text { activity** }\end{array}$ & $\begin{array}{l}\text { CMCase } \\
\text { activity }\end{array}$ & $\begin{array}{c}\text { FPase } \\
\text { activity }\end{array}$ & $\begin{array}{c}\beta- \\
\text { glucosidase } \\
\text { activity } \\
\end{array}$ & $\begin{array}{c}\text { Total } \\
\text { cellulase } \\
\text { activity } \\
\end{array}$ & $\begin{array}{l}\text { Specific } \\
\text { activity }\end{array}$ & $\begin{array}{l}\text { Xylanase } \\
\text { activity }\end{array}$ & $\begin{array}{l}\text { Specific } \\
\text { activity }\end{array}$ & $\begin{array}{c}\text { Reducing } \\
\text { sugars } \\
(\mathrm{mg} / \mathrm{g})^{* * * *}\end{array}$ & $\%$ & $\begin{array}{c}\text { Protein } \\
\text { mg/g }\end{array}$ & $\%$ & $\begin{array}{l}\text { Biodegradation } \\
\text { index }\end{array}$ \\
\hline 20 & 1.77 & 0.08 & 10.63 & 7.43 & 0.46 & 18.30 & 0.70 & 214.36 & 8.30 & 21.95 & 2.19 & 25.8 & 2.58 & 3.67 \\
\hline 25 & 2.73 & 0.10 & 11.84 & 7.44 & 0.48 & 19.50 & 0.72 & 265.31 & 9.88 & 23.85 & 2.38 & 26.85 & 2.68 & 3.87 \\
\hline 30 & 2.82 & 0.10 & 13.60 & 9.18 & 0.49 & 23.10 & 0.82 & 268.97 & 9.64 & 25.35 & 2.53 & 27.90 & 2.79 & 4.05 \\
\hline 35 & 1.35 & 0.05 & 9.19 & 5.66 & 0.45 & 15.15 & 0.57 & 115.30 & 4.36 & 24.75 & 2.47 & 26.40 & 2.64 & 3.87 \\
\hline 40 & 0.90 & 0.03 & 8.08 & 4.44 & 0.42 & 112.72 & 4.42 & 110.84 & 4.34 & 24.0 & 2.40 & 25.50 & 2.55 & 3.75 \\
\hline 45 & 0.60 & 0.02 & 7.67 & 4.39 & 0.39 & 12.39 & 0.50 & 106.89 & 4.39 & 22.80 & 2.28 & 24.30 & 2.43 & 3.57 \\
\hline $\mathrm{CD}_{0.05}$ & 0.018 & 0.018 & 0.019 & 0.018 & 0.018 & 0.015 & 0.018 & 4.274 & 0.019 & 0.019 & 0.019 & 0.022 & 0.019 & 0.021 \\
\hline S.E. & 0.008 & 0.008 & 0.008 & 0.008 & 0.008 & 0.007 & 0.008 & 1.894 & 0.008 & 0.009 & 0.009 & 0.01 & 0.009 & 0.02 \\
\hline
\end{tabular}

$* \mathrm{U} / \mathrm{g}=\mu$ moles of reducing sugars released $/ \mathrm{min} / \mathrm{g}$ of biomass

$* *$ Specific activity $=$ activity $/ \mathrm{mg}$ protein concentration

$* * *$ Reducing sugars released $\mathrm{mg} / \mathrm{g}$ 
Table2. Effect of temperature enzymes production using pretreated biomass

\begin{tabular}{|c|c|c|c|c|c|c|c|c|c|c|c|c|c|c|}
\hline \multirow{2}{*}{$\begin{array}{c}\text { Temperature } \\
\left({ }^{\circ} \mathbf{C}\right)\end{array}$} & \multicolumn{13}{|c|}{ Enzymes activities } & \multirow{2}{*}{$\begin{array}{l}\text { Biodegradation } \\
\text { Index (BI) }\end{array}$} \\
\hline & $\begin{array}{l}\text { Laccase } \\
\text { activity }\end{array}$ & $\begin{array}{l}\text { Specific } \\
\text { activity }\end{array}$ & $\begin{array}{l}\text { CMCase } \\
\text { activity }\end{array}$ & $\begin{array}{c}\text { FPase } \\
\text { activity }\end{array}$ & $\begin{array}{c}\beta- \\
\text { glucosidase } \\
\text { activity }\end{array}$ & $\begin{array}{l}\text { Cellulase } \\
\text { activity }\end{array}$ & $\begin{array}{l}\text { Specific } \\
\text { activity }\end{array}$ & $\begin{array}{c}\text { Xylanase } \\
\text { activity }\end{array}$ & $\begin{array}{l}\text { Specific } \\
\text { activity }\end{array}$ & $\begin{array}{l}\text { Reducing } \\
\text { sugars } \\
\text { (mg/g) } \\
\end{array}$ & $\%$ & $\begin{array}{c}\text { Protein } \\
\mathrm{mg} / \mathrm{g}\end{array}$ & $\%$ & \\
\hline 20 & 2.01 & 0.12 & 10.25 & 6.96 & 0.46 & 17.55 & 1.04 & 162.99 & 9.74 & 21.79 & 2.17 & 16.72 & 1.67 & 2.75 \\
\hline 25 & 2.58 & 0.09 & 11.20 & 7.23 & 0.47 & 18.75 & 0.66 & 263.42 & 9.29 & 22.35 & 2.23 & 28.30 & 2.83 & 3.94 \\
\hline 30 & 2.72 & 0.09 & 12.30 & 8.28 & 0.49 & 20.70 & 0.71 & 265.63 & 9.22 & 24.90 & 2.49 & 28.8 & 2.88 & 4.12 \\
\hline 35 & 0.85 & 0.03 & 9.12 & 5.43 & 0.45 & 14.85 & 0.54 & 127.97 & 4.66 & 24.45 & 2.44 & 27.45 & 2.74 & 3.96 \\
\hline 40 & 0.23 & 0.008 & 8.06 & 5 & 0.4 & 13.50 & 0.51 & 117.25 & 4.44 & 23.55 & 2.35 & 26.4 & 2.64 & 3.81 \\
\hline 45 & 0.13 & 0.005 & 7.73 & 4.36 & 0.41 & 12.30 & 0.48 & 97.65 & 3.82 & 22.50 & 2.25 & 25.5 & 2.55 & 3.67 \\
\hline $\mathrm{CD}_{0.05}$ & 0.017 & 0.016 & 0.018 & 0.019 & 0.018 & 0.019 & 0.018 & 0.018 & 0.019 & 0.02 & 0.019 & 0.018 & 0.019 & 0.021 \\
\hline S.E. & 0.008 & 0.007 & 0.008 & 0.006 & 0.006 & 0.008 & 0.008 & 0.006 & 0.008 & 0.009 & 0.008 & 0.008 & 0.009 & 0.02 \\
\hline
\end{tabular}


Table3. Effect of substrate: moisture level on enzymes production using untreated biomass

\begin{tabular}{|c|c|c|c|c|c|c|c|c|c|c|c|c|c|c|}
\hline \multirow{2}{*}{$\begin{array}{c}\text { Substrate } \\
\text { :moisture } \\
\text { ratio }\end{array}$} & \multicolumn{9}{|c|}{ Enzyme activities } & \multicolumn{2}{|c|}{ Reducing sugars } & \multicolumn{2}{|c|}{ Protein } & \multirow{2}{*}{$\begin{array}{l}\text { Biodegradation } \\
\text { Index (BI) }\end{array}$} \\
\hline & $\begin{array}{c}\text { Laccase } \\
(\mathrm{U} / \mathrm{g})\end{array}$ & $\begin{array}{l}\text { Specific } \\
\text { activity } \\
\text { (U/mg) }\end{array}$ & $\begin{array}{c}\text { CMCase } \\
\text { (U/g) }\end{array}$ & $\begin{array}{l}\text { FPase } \\
(\mathbf{U} / \mathrm{g})\end{array}$ & $\begin{array}{c}\beta- \\
\text { glucosidase } \\
(\mathrm{U} / \mathrm{g})\end{array}$ & $\begin{array}{c}\text { Cellulase } \\
\text { activity } \\
\text { (U/g) }\end{array}$ & $\begin{array}{l}\text { Specific } \\
\text { activity } \\
\text { (U/mg) }\end{array}$ & $\begin{array}{c}\text { Xylanase } \\
(\mathbf{U} / \mathrm{g})\end{array}$ & $\begin{array}{l}\text { Specific } \\
\text { activity } \\
\text { (U/mg) }\end{array}$ & $(\mathrm{mg} / \mathrm{g})$ & $\%$ & $\mathrm{mg} / \mathrm{g}$ & $\%$ & \\
\hline 01:02 & 1.37 & 0.06 & 10.93 & 11.14 & 1.36 & 23.25 & 1.04 & 252.75 & 11.36 & 22.8 & 2.28 & 22.23 & 2.22 & 3.36 \\
\hline 01:03 & 3.07 & 0.09 & 12.39 & 13.05 & 1.39 & 26.70 & 0.82 & 262.80 & 8.07 & 24.45 & 2.44 & 32.53 & 3.25 & 4.47 \\
\hline 01:04 & 3.45 & 0.10 & 13.83 & 12.30 & 1.83 & 27.90 & 0.85 & 279.30 & 8.54 & 26.10 & 2.61 & 32.70 & 3.27 & 4.57 \\
\hline 01:05 & 2.02 & 0.06 & 9.13 & 13. & 1.62 & 23.85 & 0.75 & 254.70 & 8.06 & 2 & 2.58 & 31.59 & 3.15 & 4.44 \\
\hline 01:06 & 0.89 & 0.03 & 6.09 & 9.43 & 1.45 & 20.40 & 0.71 & 234.75 & 8.28 & 25.50 & 2.55 & 28.35 & 2.83 & 4.10 \\
\hline $\mathrm{CD}_{0.05}$ & 0.018 & 0.017 & 0.019 & 0.044 & 0.018 & 0.015 & 0.018 & 0.044 & 0.018 & 0.019 & 0.019 & 0.022 & 0.019 & 0.022 \\
\hline S.E & 0.008 & 0.008 & 0.009 & 0.02 & 0.008 & 0.007 & 0.008 & 0.019 & 0.008 & 0.009 & 0.008 & 0.01 & 0.008 & 0.01 \\
\hline
\end{tabular}


Table4. Effect of substrate: moisture level enzymes production using pretreated biomass

\begin{tabular}{|c|c|c|c|c|c|c|c|c|c|c|c|c|c|c|}
\hline \multirow{2}{*}{$\begin{array}{c}\text { Substrate } \\
\text { :moisture } \\
\text { ratio }\end{array}$} & \multicolumn{9}{|c|}{ Enzyme activities } & \multicolumn{2}{|c|}{ Reducing sugars } & \multicolumn{2}{|c|}{ Protein } & \multirow{2}{*}{$\begin{array}{l}\text { Biodegradation } \\
\text { Index (BI) }\end{array}$} \\
\hline & $\begin{array}{c}\text { Laccase } \\
(\mathbf{U} / \mathbf{g})\end{array}$ & $\begin{array}{c}\text { Specific } \\
\text { activity } \\
(\mathrm{U} / \mathrm{mg})^{* *}\end{array}$ & $\begin{array}{c}\text { CMCase } \\
(\mathbf{U} / \mathrm{g})\end{array}$ & $\begin{array}{c}\text { FPase } \\
(\mathrm{U} / \mathrm{g})\end{array}$ & $\begin{array}{c}\boldsymbol{\beta}- \\
\text { glucosidase } \\
(\mathrm{U} / \mathrm{g})\end{array}$ & $\begin{array}{c}\text { Cellulase } \\
\text { activity } \\
\text { (U/g) }\end{array}$ & $\begin{array}{l}\text { Specific } \\
\text { activity } \\
\text { (U/mg) }\end{array}$ & $\begin{array}{c}\text { Xylanase } \\
(\mathrm{U} / \mathrm{g})\end{array}$ & $\begin{array}{l}\text { Specific } \\
\text { activity } \\
\text { (U/mg) }\end{array}$ & $(\mathrm{mg} / \mathrm{g})^{* * * *}$ & $\%$ & $\mathrm{mg} / \mathrm{g}$ & $\%$ & \\
\hline 01:02 & 0.39 & 0.01 & 10.65 & 10.12 & 1.25 & 21.90 & 1.08 & 228.45 & 11.32 & 24.83 & 2.48 & 20.18 & 2.01 & 3.25 \\
\hline 01:03 & 1.90 & 0.08 & $\begin{array}{l}11.25 \\
(0.75)\end{array}$ & 10.39 & 1.44 & 22.95 & 1.02 & 253.35 & 133 & 31.27 & 3.12 & 22.35 & 2.23 & 3.79 \\
\hline 01:04 & 3.16 & 0.12 & 12.75 & 12 . & 1.83 & 27.00 & 1.04 & 277.95 & 10.77 & 32.65 & 3.26 & 25.80 & 2.58 & 4.21 \\
\hline $01: 05$ & 0.94 & 0.03 & 13.33 & 9.67 & 1.57 & 24.75 & 0.97 & 258.45 & 7.31 & 31.48 & 3.14 & 25.35 & 2.53 & 4.10 \\
\hline 01:06 & 0.70 & 0.03 & 8.55 & 8.73 & 1.53 & 18.75 & 0.80 & 228.15 & 9.81 & 27.79 & 2.77 & 23.25 & 2.32 & 3.70 \\
\hline $\mathrm{CD}_{0.05}$ & 0.018 & 0.018 & 0.021 & 0.018 & 0.018 & 0.019 & 0.018 & 0.374 & 0.018 & 0.024 & 0.018 & 0.019 & 0.019 & 0.024 \\
\hline S.E & 0.008 & 0.008 & 0.009 & 0.008 & 0.008 & 0.009 & 0.008 & 0.166 & 0.008 & 0.011 & 0.008 & 0.008 & 0.009 & 0.02 \\
\hline
\end{tabular}


Table5. Effect of incubation time on enzymes production using untreated biomass

\begin{tabular}{|c|c|c|c|c|c|c|c|c|c|c|c|c|c|c|}
\hline \multirow{2}{*}{$\begin{array}{l}\text { Incubation } \\
\text { time (days) }\end{array}$} & \multicolumn{9}{|c|}{ Enzyme activities } & \multicolumn{2}{|c|}{ Reducing sugars } & \multicolumn{2}{|c|}{ Protein } & \multirow{2}{*}{$\begin{array}{l}\text { Biodegradation } \\
\text { index (BI) }\end{array}$} \\
\hline & $\begin{array}{c}\text { Laccase } \\
\text { (U/g) }\end{array}$ & $\begin{array}{l}\text { Specific } \\
\text { activity } \\
\text { (U/mg) } \\
\end{array}$ & CMCase & $\begin{array}{c}\text { FPase } \\
\text { activity }\end{array}$ & $\begin{array}{c}\beta- \\
\text { glucosidase } \\
\text { activity } \\
\end{array}$ & $\begin{array}{c}\text { Cellulase } \\
\text { Activity } \\
\text { (U/g) } \\
\end{array}$ & $\begin{array}{l}\text { Specific } \\
\text { activity }\end{array}$ & $\begin{array}{c}\text { Xylanase } \\
\text { activity } \\
\text { (U/g) } \\
\end{array}$ & $\begin{array}{l}\text { Specific } \\
\text { activity }\end{array}$ & $(\mathrm{mg} / \mathrm{g})$ & $\%$ & $(\mathrm{mg} / \mathrm{g})$ & $\%$ & \\
\hline 3 & 0.27 & 0.01 & 10.95 & 10.05 & 0.44 & 21.30 & 0.91 & 253.50 & 10.83 & 28.35 & 2.83 & 23.40 & 2.34 & 3.75 \\
\hline 5 & 1.68 & 0.06 & 11.7 & 10.50 & 0.46 & 22.65 & 0.93 & 254.10 & .45 & 31.80 & 3.18 & 24.30 & 2.43 & 4.02 \\
\hline 7 & 2.54 & 0.10 & 14.85 & 13. & 0.51 & 29.25 & 1.16 & 282.75 & 11.15 & 36.75 & 3.67 & 25.35 & 2.53 & 4.36 \\
\hline 10 & 3.45 & 0.12 & 13.80 & 10.35 & 0.55 & 24.60 & 0.91 & 293.10 & 10.91 & 33.45 & 3.34 & 26.85 & 2.68 & 4.35 \\
\hline 12 & 0.30 & 0.01 & 9.30 & 10.80 & 0.30 & 20.4 & 0.83 & 278.85 & 11.47 & 29.40 & 2.94 & 24.3 & 2.43 & 3.90 \\
\hline $\mathrm{CD}_{0.05}$ & 0.018 & 0.019 & 0.018 & 0.018 & 0.018 & 0.019 & 0.018 & 0.064 & 0.003 & 0.019 & 0.019 & 0.018 & 0.019 & 0.023 \\
\hline S.E. & 0.008 & 0.006 & 0.008 & 0.006 & 0.008 & 0.006 & 0.008 & 0.028 & 0.001 & 0.009 & 0.008 & 0.008 & 0.009 & 0.016 \\
\hline
\end{tabular}


Table6. Effect of incubation time enzymes production using pretreated biomass

\begin{tabular}{|c|c|c|c|c|c|c|c|c|c|c|c|c|c|c|}
\hline \multirow{2}{*}{$\begin{array}{l}\text { Incubation } \\
\text { time (days) }\end{array}$} & \multicolumn{9}{|c|}{ Enzyme activities } & \multicolumn{2}{|c|}{ Reducing sugars } & \multicolumn{2}{|c|}{ Protein } & \multirow{2}{*}{$\begin{array}{c}\text { Biodegradation } \\
\text { index }(\mathrm{BI})\end{array}$} \\
\hline & $\begin{array}{c}\text { Laccase } \\
(\mathrm{U} / \mathrm{g})\end{array}$ & $\begin{array}{l}\text { Specific } \\
\text { activity } \\
\text { (U/mg) }\end{array}$ & $\begin{array}{c}\text { CMCase } \\
\text { activity }\end{array}$ & $\begin{array}{c}\text { FPase } \\
\text { activity }\end{array}$ & $\begin{array}{c}\beta- \\
\text { glucosidase } \\
\text { activity }\end{array}$ & $\begin{array}{c}\text { Total } \\
\text { cellulase } \\
\text { activity } \\
\end{array}$ & $\begin{array}{l}\text { Specific } \\
\text { activity }\end{array}$ & $\begin{array}{c}\text { Xylanase } \\
\text { activity }\end{array}$ & $\begin{array}{l}\text { Specific } \\
\text { activity }\end{array}$ & $(\mathrm{mg} / \mathrm{g})$ & $\%$ & $(\mathrm{mg} / \mathrm{g})$ & $\%$ & \\
\hline 3 & 0.66 & 0.02 & 10.80 & 9.45 & 0.41 & 20.55 & 0.86 & 239.70 & 10.05 & 25.25 & 2.52 & 23.85 & 2.38 & 3.64 \\
\hline 5 & 1.64 & 0.06 & 11.40 & 9.75 & 0.46 & 21.60 & 0.88 & 243.45 & 10.01 & 28.35 & 2.83 & 24.3 & 2.43 & 3.84 \\
\hline 7 & 1.75 & 0.06 & 13.35 & 13.35 & 0.56 & 27.15 & 0.97 & 288.30 & 10.38 & 36.60 & 3.66 & 27.75 & 2.77 & 4.60 \\
\hline 10 & 3.30 & 0.11 & 13.20 & 9.90 & 0.55 & 23.55 & 0.78 & 289.50 & 9.69 & 33.00 & 3.30 & 29.85 & 2.98 & 4.63 \\
\hline 12 & 0.30 & 0.01 & 9.45 & 9.30 & 0.45 & 19.20 & 0.67 & 227.25 & 0.96 & 29.85 & 2.98 & 28.35 & 2.83 & 4.32 \\
\hline $\mathrm{CD}_{0.05}$ & 0.018 & 0.016 & 0.018 & 0.018 & 0.019 & 0.018 & 0.018 & 0.038 & 0.018 & 0.017 & 0.018 & 0.044 & 0.018 & 0.019 \\
\hline S.E. & 0.008 & 0.007 & 0.008 & 0.008 & 0.009 & 0.007 & 0.008 & 0.017 & 0.008 & 0.008 & $0 . .008$ & 0.019 & 0.008 & 0.009 \\
\hline
\end{tabular}


Table 7. Effect of pH on enzymes production using untreated biomass

\begin{tabular}{|c|c|c|c|c|c|c|c|c|c|c|c|c|c|c|}
\hline \multirow[t]{2}{*}{$\mathbf{p H}$} & \multicolumn{9}{|c|}{ Enzyme activities } & \multicolumn{2}{|c|}{ Reducing sugars } & \multicolumn{2}{|c|}{ Protein } & \multirow{2}{*}{$\begin{array}{c}\text { Biodegradation } \\
\text { Index (BI) }\end{array}$} \\
\hline & $\begin{array}{c}\text { Laccase } \\
(\mathrm{U} / \mathrm{g})\end{array}$ & $\begin{array}{l}\text { Specific } \\
\text { activity } \\
\text { (U/mg) }\end{array}$ & $\begin{array}{l}\text { CMCase } \\
\text { activity }\end{array}$ & $\begin{array}{c}\text { FPase } \\
\text { activity }\end{array}$ & $\begin{array}{c}\beta- \\
\text { glucosidase } \\
\text { activity } \\
\end{array}$ & $\begin{array}{c}\text { Total } \\
\text { cellulase } \\
\text { activity } \\
\end{array}$ & $\begin{array}{l}\text { Specific } \\
\text { activity }\end{array}$ & $\begin{array}{c}\text { Xylanase } \\
\text { activity }\end{array}$ & $\begin{array}{l}\text { Specific } \\
\text { activity }\end{array}$ & $(\mathrm{mg} / \mathrm{g})$ & $\%$ & $\mathrm{mg} / \mathrm{g}$ & $\%$ & \\
\hline 4.5 & 1.80 & 0.07 & 11.40 & 9.75 & 0.42 & 21.45 & 0.87 & 244.41 & 9.9 & 28.80 & 2.88 & 24.60 & 2.46 & 3.90 \\
\hline 5 & 2.75 & 0.10 & 11.85 & 10.80 & 0.45 & 23.10 & 0.91 & 257.85 & 10.23 & 29.85 & 2.98 & 25.20 & 2.52 & 4.01 \\
\hline 5.5 & 3.45 & 0.13 & 14.70 & 14.40 & 0.60 & 29.70 & 1.14 & 350.17 & 13.49 & 36.90 & 3.69 & 25.95 & 2.59 & 4.43 \\
\hline 6 & 3.75 & 0.14 & 13.80 & 13.20 & 0.56 & 27.45 & 1.03 & 235.50 & 8.92 & 33.90 & 3.39 & 26.40 & 2.64 & 4.33 \\
\hline 6.5 & 2.85 & 0.11 & 13.20 & 10.95 & 0.45 & 24.60 & 0.97 & 209.26 & 8.25 & 31.95 & 3.19 & 25.35 & 2.53 & 4.12 \\
\hline $\mathrm{CD}_{0.05}$ & 0.019 & 0.017 & 0.018 & 0.018 & 0.018 & 0.019 & 0.016 & 0.021 & 0.018 & 0.022 & 0.019 & 0.019 & 0.019 & 0.023 \\
\hline S.E. & 0.009 & 0.006 & 0.008 & 0.006 & 0.008 & 0.009 & 0.007 & 0.01 & 0.008 & 0.01 & 0.009 & 0.008 & 0.008 & 0.013 \\
\hline
\end{tabular}


Table8. Effect of pH enzymes production by using pretreated biomass

\begin{tabular}{|c|c|c|c|c|c|c|c|c|c|c|c|c|c|c|}
\hline \multirow[t]{2}{*}{ pH } & \multicolumn{9}{|c|}{ Enzyme activities } & \multicolumn{2}{|c|}{ Reducing sugars } & \multicolumn{2}{|c|}{ Protein } & \multirow{2}{*}{$\begin{array}{l}\text { Biodegradation } \\
\text { Index (BI) }\end{array}$} \\
\hline & $\begin{array}{c}\text { Laccase } \\
(\mathrm{U} / \mathrm{g})\end{array}$ & $\begin{array}{l}\text { Specific } \\
\text { activity } \\
\text { (U/mg) }\end{array}$ & $\begin{array}{l}\text { CMCase } \\
\text { activity }\end{array}$ & $\begin{array}{c}\text { FPase } \\
\text { activity }\end{array}$ & $\begin{array}{c}\beta- \\
\text { glucosidase } \\
\text { activity }\end{array}$ & $\begin{array}{c}\text { Total } \\
\text { cellulase } \\
\text { activity }\end{array}$ & $\begin{array}{l}\text { Specific } \\
\text { activity }\end{array}$ & $\begin{array}{l}\text { Xylanase } \\
\text { activity }\end{array}$ & $\begin{array}{l}\text { Specific } \\
\text { activity }\end{array}$ & $(\mathrm{mg} / \mathrm{g}) * * *$ & $\%$ & $\mathrm{mg} / \mathrm{g}$ & $\%$ & \\
\hline 4.5 & 1.50 & 0.06 & 11.25 & 9.30 & 0.30 & 20.85 & 0.84 & 254.85 & 10.29 & 28.20 & 2.82 & 24.75 & 2.47 & 3.88 \\
\hline 5 & 2.70 & 0.10 & 12.00 & 10.35 & 0.45 & 22.80 & 0.89 & 255.45 & 10.07 & 28.50 & 2.85 & 25.35 & 2.53 & 3.95 \\
\hline 5.5 & 3.45 & 0.13 & 12.30 & 12.30 & 0.52 & 25.05 & 0.97 & 335.25 & 12.99 & 32.10 & 3.21 & 25.80 & 2.58 & 4.18 \\
\hline 6 & 3.60 & 0.14 & 13.50 & 14.40 & 0.49 & 28.20 & 1.13 & 301.80 & 12.12 & 31.80 & 3.18 & 24.90 & 2.49 & 4.08 \\
\hline 6.5 & 2.85 & 0.11 & 11.85 & 10.80 & 0.42 & 22.95 & 0.95 & 234.90 & 9.78 & 29.70 & 2.97 & 24.00 & 24.00 & 3.88 \\
\hline $\mathrm{CD}_{0.05}$ & 0.018 & 0.018 & 0.018 & 0.018 & 0.018 & 0.016 & 0.018 & 0.076 & 0.019 & 0.017 & 0.018 & 0.02 & 0.019 & 0.024 \\
\hline S.E. & 0.008 & 0.006 & 0.006 & 0.008 & 0.008 & 0.007 & 0.008 & 0.034 & 0.009 & 0.007 & 0.008 & 0.009 & 0.009 & 0.014 \\
\hline
\end{tabular}




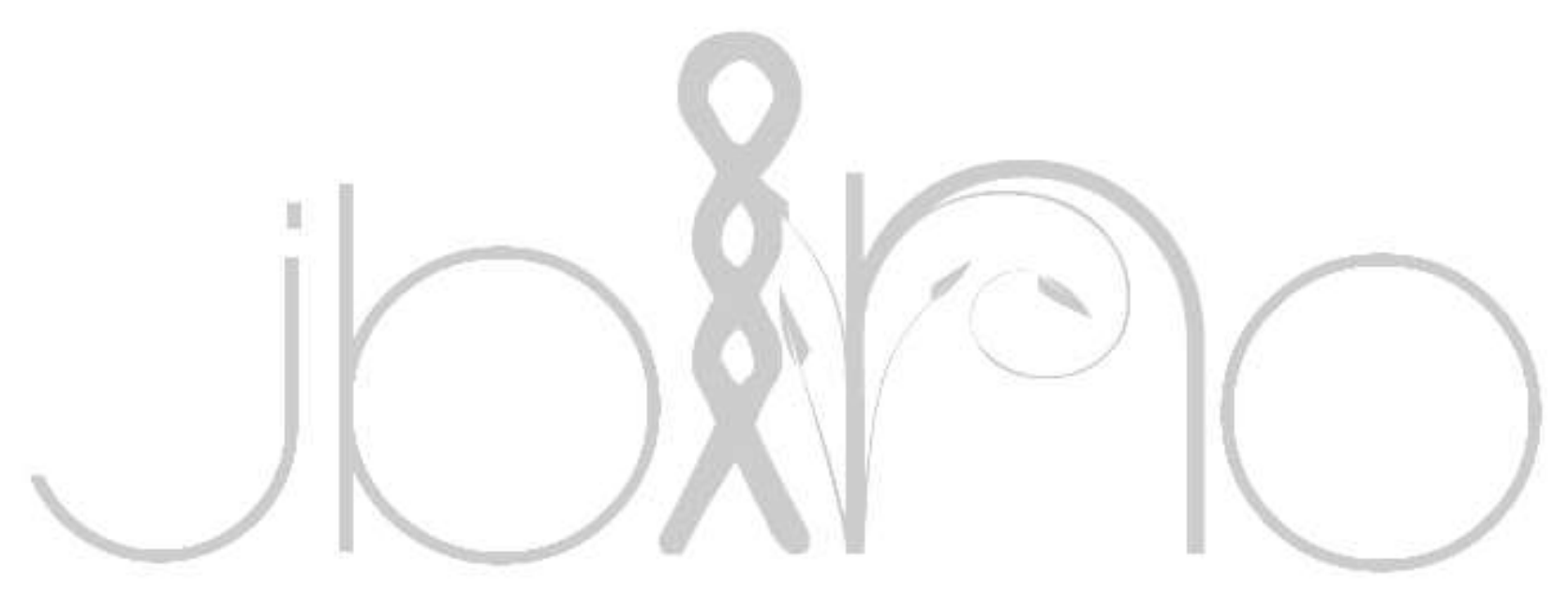

2020 November Edition | www.jbino.com | Innovative Association colo 


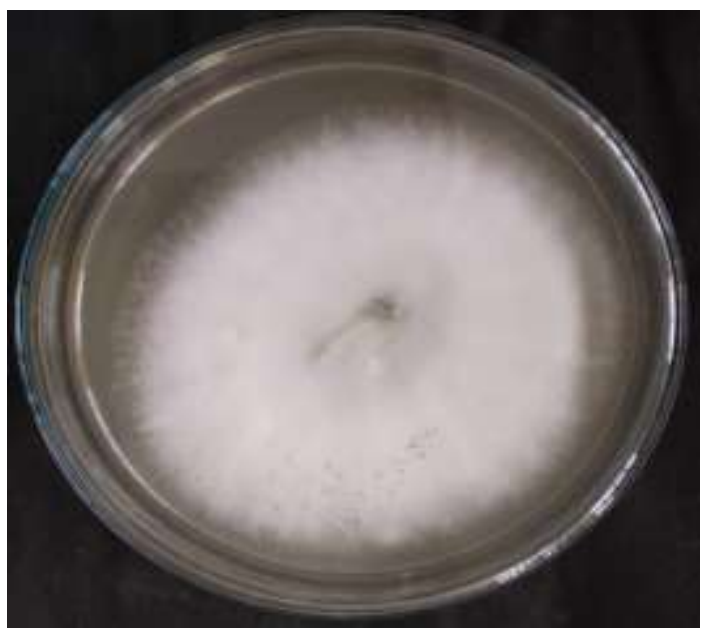

Fig1. Morphology of Trichoderma sp. SH2

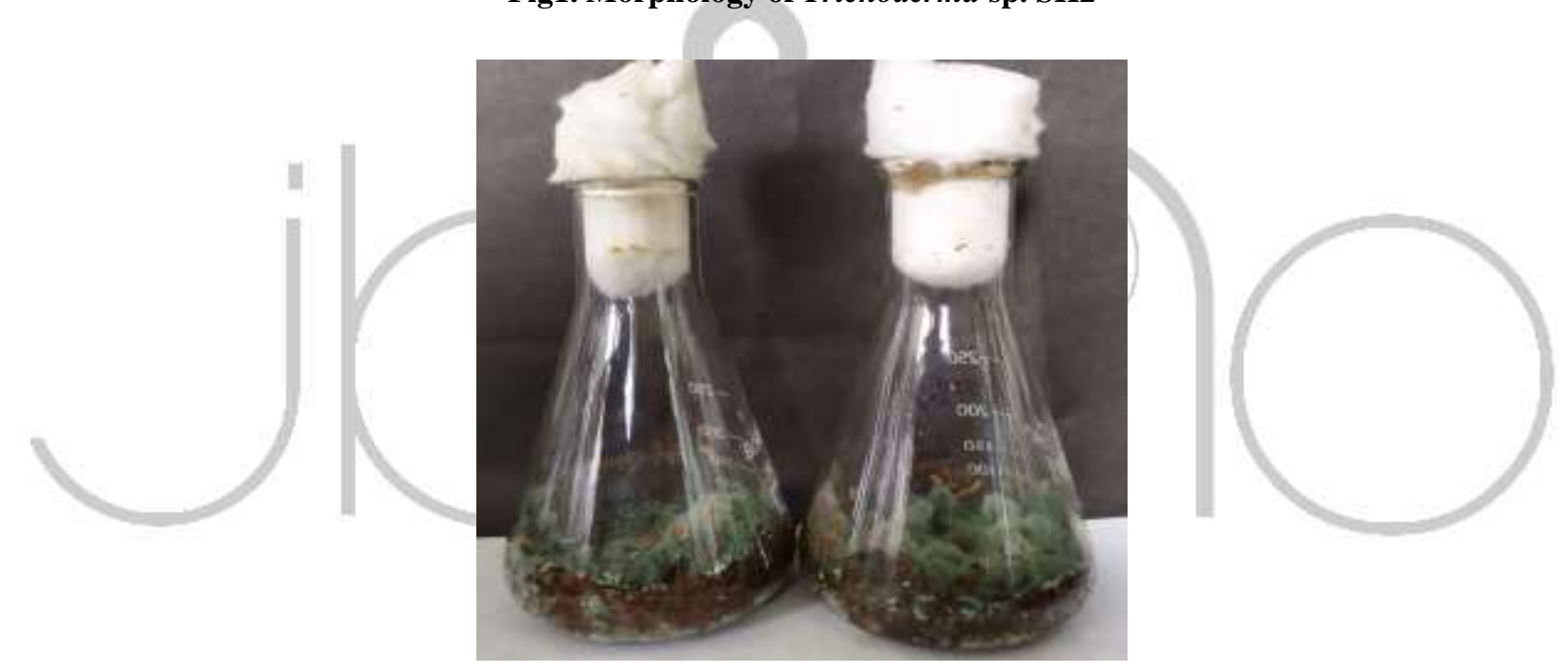

Fig2. Enzyme production by Trichoderma sp. SH2 under solid state fermentation 


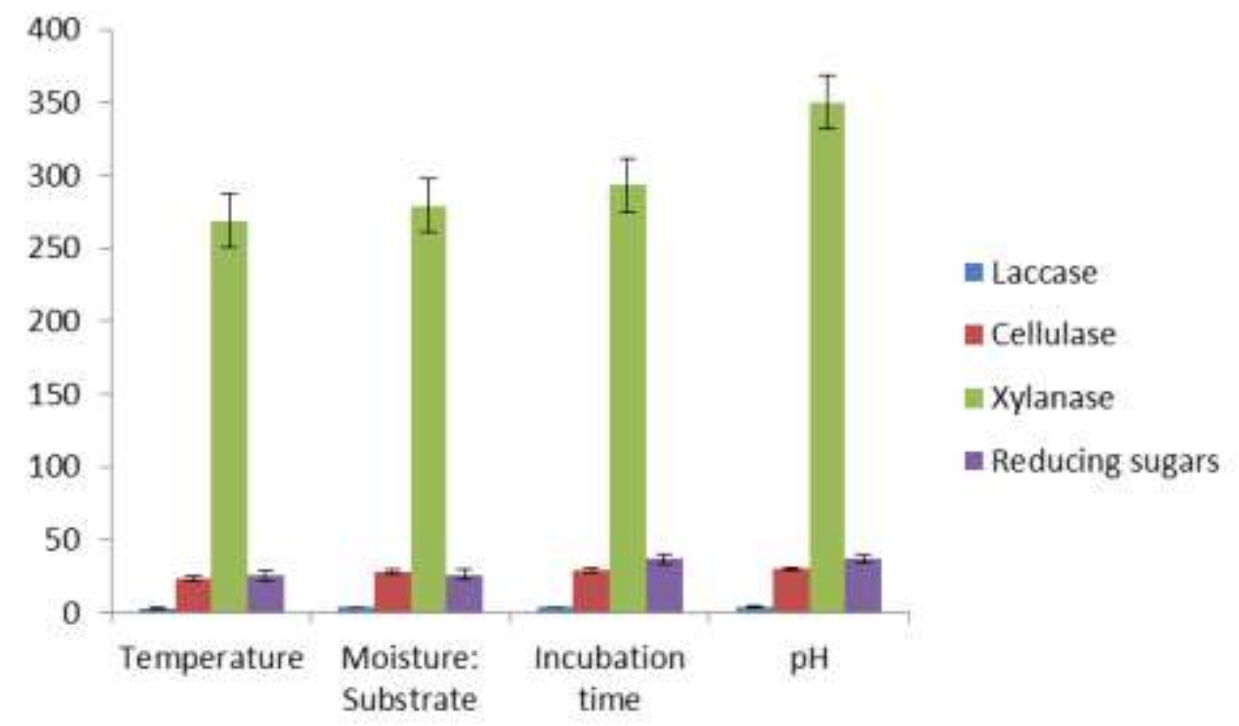

Fig3. Overall increase in enzyme activities and reducing sugars using untreated biomass after optimization of process parameters

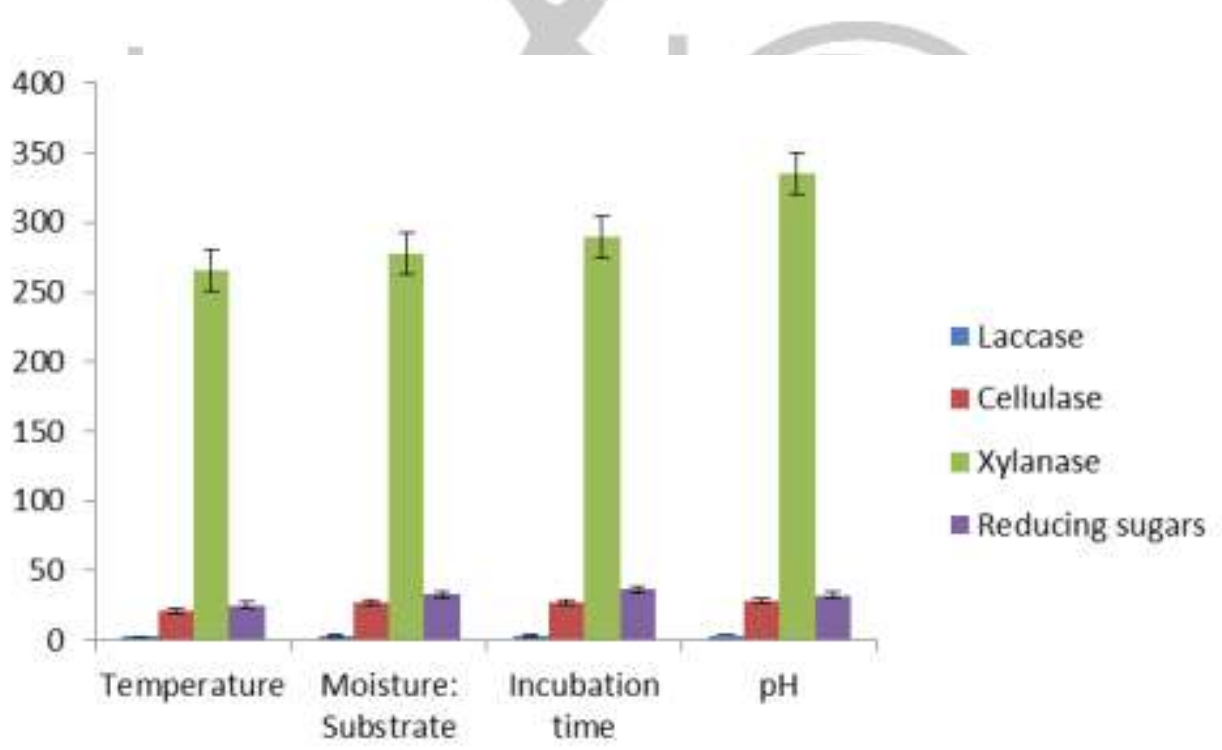

Fig4. Overall increase in enzyme activities and reducing sugars using pretreated biomass after optimization of process parameters 


\section{Conclusion}

The demand for enzymes in the global market is projected to rise at a fast pace in recent years. There has been a great increase in industrial applications of hydrolytic enzymes owing to their significant biotechnological uses. Therefore the study was undertaken with aims of enhanced production of laccase, cellulase and xylanase enzymes from a potential fungal strain Trichoderma sp. SH2. In the present study, an attempt was made to optimize the various parameters i.e. different moisture: substrate ratio, temperature, incubation days and $\mathrm{pH}$ to increase production level of enzymes and reducing sugars and achieved 75.20 (laccase), 77.77 (cellulase), 76.81 (xylanase) and 68.69 (reducing sugars) \% increase respectively from Trichoderma sp. SH2.

\section{Acknowledgement}

Authors gratefully acknowledge the financial support given by National Mission on Himalayan studies (NMHS), Ministry of Environment, Forest and Climate Change (MoEF\&CC), Govt of India, New Delhi and G.B. Pant National Institute of Himalayan Environment and Sustainable Development (GBPNIHESD), KosiKataramal, Almora, Uttarakhand.

Conflict of Interest: There is no conflict of interest.

\section{References}

Acharya D K, Chabhadiya S B, Shah J H, Shilpkar P, Acharya P B and Modi H A.
2010. Enzyme profiling of lignocellulolytic fungi. International Journal of Biological and Chemical Sciences. 4:443-449.

Barakat A, Mayer C, Solhy A, Arancon R A and De Vries H. 2014. Mechanical pretreatments of lignocellulosic biomass: towards facile and environmentally sound technologies for biofuels production. Royal Society of Chemistry and Advances. 4:109127.

Berghem L.E.R., Petterson L.G. 1973. Mechanism of enzymatic cellulose degradation and purification of a cellulolytic enzyme from $T$. viride active on highly ordered cellulose. Journal of Biochemistry. 37: 21-30.

Bollag D.M. and Edelstein S.J. 1991. Concentrating protein solutions In: Protein methods. A John Wiley Sons, INC, Publications, USA. p.45.

Elisashvili V, Kachlishvili E. 2009. Physiological regulation of laccase and manganese peroxidase production by white-rot basidiomycetes. Journal of Biotechnology. 144:37-42.

Li S, Yang X, Yang S. 2012. Technology prospecting on enzymes: application, marketing and engineering. Comput Struct Biotechnol J. 2:1-11

Lowry O. H. Rosebrough N. J., Farr A. L. and Randall R. J. 1951. Protein measurement with the Folin-phenol reagent. Journal of Biological Chemistry. 193:265-75. 
Massadeh M I, Yusoff W M W, Omar O and Kader J. 2001. Synergism of cellulase enzymes in mixed culture solid state fermentation. Biotechnology Letters. 23 (21): 1771-1774.

Miller GL. 1959. Use of dinitrosalicylic acid reagent for determination of reducing sugars. Anal Chem 31:426-428.

Mishra P K, Wimmer R and Kumar D. 2016. Aerosol assisted self-assembly as a route to synthesize solid and hollow spherical lignin colloids and its utilization in layer by layer deposition. Ultrason Sonochemistry. 35: 4050.

Nagraj A K, Singhvi M A, Kumar $V R$ and Gokhale D A. 2014. Optimization studies for enhancing cellulase production by Penicillium janthinellum mutant EU2D-21 using response surface methodology. Bioresources. 9:1914-1923.

Reese E T, Mandel M. 1963. Enzymatic hydrolysis of cellulose and its derivatives In: Method Carbohydrate Chemistry (Whistles
R L eds.). Academic Press London. pp. 139143.

Rehan A M, Hassan $E$ and Ramadan E.2016. Production of laccase enzyme for their potential application to decolorize fungal pigments on aging paper and parchment. Annals of Agricultural Sciences. 61:1-11.

Sharma N and Sharma N. 2016. Cellulase production and optimization of process parameters by one factor at a time technique from mutant strain of Bacillus stratosphericus $\mathrm{N} 12$ (M) and its scale up in a stirred tank bioreactor. World Journal of Pharmacy and Pharmaceutical Sciences. 5 (2): 1459-1472.

Sharma N, Sharma N and Dimple. 2019. An Evaluation Study of Different White Rot Fungi for Degradation of Pine Needles under Solid State Fermentation. International Journal of Current Microbiology and Applied Sciences. 8:588560. 